Research Paper

\title{
Upregulation of Heat Shock Proteins (HSPA12A, HSP90B1, HSPA4, HSPA5 and HSPA6) in Tumour Tissues Is Associated with Poor Outcomes from HBV-Related Early-Stage Hepatocellular Carcinoma
}

\author{
Zongguo Yang1 ${ }^{*}$, Liping Zhuang $2,3^{*}$, Peter Szatmary4,5, Li Wen ${ }^{4,5}$, Hua Sun ${ }^{1}$, Yunfei Lu1 ${ }^{1}$ Qingnian Xu1, \\ Xiaorong Chen ${ }^{1 凶}$ \\ 1. Department of Traditional Chinese Medicine, Shanghai Public Health Clinical Center, Fudan University, Shanghai 201508, China; \\ 2. Department of Integrative Medicine, Fudan University Shanghai Cancer Center, Shanghai 200032, China; \\ 3. Department of Oncology, Shanghai Medical College, Fudan University, Shanghai 200032, China. \\ 4. NIHR Liverpool Pancreas Biomedical Research Unit, Royal Liverpool University Hospital, Liverpool L69 3GA, UK. \\ 5. Department of Molecular and Clinical Cancer Medicine, Institute of Translation medicine, University of Liverpool, Liverpool L69 3GA, \\ UK.
}

*Equal contributors.

$\square$ Corresponding author: Xiaorong Chen, Shanghai Public Health Clinical Center, Fudan University, 2901 Caolang Road, Jinshan District, Shanghai 201508, China. Tel: +86 21 37990333; Fax: +86 21 57248762; Email: xiaorong3chen@163.com.

(c) 2015 Ivyspring International Publisher. Reproduction is permitted for personal, noncommercial use, provided that the article is in whole, unmodified, and properly cited. See http://ivyspring.com/terms for terms and conditions.

Received: 2014.10.06; Accepted: 2015.01.21; Published: 2015.02.15

\begin{abstract}
Background: Heat shock proteins (HSPs) are overexpressed in human hepatocellular carcinoma (HCC) tissue and correlate with aggressiveness and prognosis of HCC.

Methods: Using the GSE14520 microarray expression profile from Gene Expression Omnibus, we compared HSP gene expression between tumour and non-tumour tissues and correlated this with outcomes in HCC patients.

Results: We analysed 220 hepatitis B virus (HBV)-related HCC patients and $25 \mathrm{HSPs}$ in this study. With the exception of HSPA4L, HSPA12A and HSPB8, members of the HSP family, including HSPH1, HSPBPI, HSPA1A, HSPA1B, HSPAIL, HSPA2, HSPA4, HSPA5, HSPA8, HSPA9, HSPAA1, HSPAB1, HSPA 14, HSPB1 1, HSPA13, HSP90B1 and HSPBAP1, were all overexpressed in tumour tissues (all $P<0.001$ ). In contrast, HSPB6, HSPB7, HSPA6, HSPB2 and HSPB3 were upregulated in non-tumour tissues (all $P<0.001$ ). Multivariate analysis showed that cirrhosis $(\mathrm{HR}=5.282,95 \% \mathrm{Cl}$ $=1.294-21.555, P=0.02)$, Barcelona Clinic liver cancer $(B C L C)$ staging $(H R=2.151,95 \% \mathrm{Cl}=$ $1.682-2.750, P<0.001)$, HSPA $12 \mathrm{~A}(\mathrm{HR}=1.042,95 \% \mathrm{Cl}=1.003-1.082, P=0.033)$ and HSP90B1 $(\mathrm{HR}=1.001,95 \% \mathrm{Cl}=1.000-1.001, P=0.011)$ were negatively associated with survival of $\mathrm{HBV}$-related $\mathrm{HCC}$ patients. Furthermore, advanced $\mathrm{BCLC}$ staging $(\mathrm{HR}=1.797,95 \% \mathrm{Cl}=$ 1.439-2.244, $P<0.001$ ) was also associated with earlier recurrence of $\mathrm{HCC}$. The high expression of HSPA4 (HR $=1.002,95 \% \mathrm{Cl}=1.000-1.004, P=0.019)$, HSPA5 $(\mathrm{HR}=1.0,95 \% \mathrm{Cl}=1.0-1.0, P$ $=0.046)$ and HSPA6 $(\mathrm{HR}=1.008,95 \% \mathrm{Cl}=1.001-1.015, P=0.021)$ was similarly associated with $\mathrm{HCC}$ recurrence.

Conclusions: The expression of most HSPs was higher in tumour tissues than in non-tumour tissues. High BCLC staging scores, advanced cirrhosis and the overexpression of HSPA12A and HSP90B1 might be associated with poor survival from HCC, whereas high levels of HSPA4, HSPA5 and HSPA6 might be associated with earlier recurrence of HCC.
\end{abstract}

Key words: heat shock proteins; carcinoma, hepatocellular; survival; recurrence; BCLC stage; cirrhosis; HSPA12A; HSP90B1; HSPA4; HSPA5; HSPA6; HSP70. 


\section{Introduction}

Globally, hepatocellular carcinoma (HCC) is the most common primary liver cancer, the fifth most common cancer and the third most common cause of cancer-related deaths $[1,2]$. Owing to changes in the prevalence of the two major risk factors, hepatitis $\mathrm{B}$ virus (HBV) and hepatitis $C$ virus, its overall incidence remains alarmingly high in the developing world and is steadily rising across most of the developed world. Currently, there are an estimated 300 million carriers of $\mathrm{HBV}$ in the world, and as many as $25 \%$ may develop HCC $[3,4]$. The widespread search for effective biomarkers of HCC using promising novel proteomic techniques is hoped to lead to earlier diagnosis and improve prognosis by allowing earlier intervention.

Heat shock proteins (HSPs) are ubiquitous molecules within cells that act as molecular chaperones in conditions of stress, including carcinogenesis. As apoptosis inhibitors, HSPs are overexpressed in human HCC tissues and correlate with its aggressiveness and prognosis [5]. Previous studies have found that a set of highly abundant HSPs on the cell surface of tumours, including HSP60, HSP70, HSP90 and HSP27. An increase in intracellular concentrations of HSPs is closely related to the genesis and development of HCC and is a useful marker of progression and aggravation of HCC [5-8]. There is good correlation between the expression of HSPs and the resistance of cancer cells to chemotherapy. The inhibition of HSP90, HSP70 and/or HSP27 is thus emerging as a novel strategy for cancer therapy. For example, HSPs can be included in vaccination protocols, due to their function as chaperones of peptide antigens $[9$, 10].

An investigation of protein expression profiles for $67 \mathrm{HCC}$ cases and 12 healthy subjects by Luk et al. found that HSP70, HSP27 and a glucose-regulated protein (GRP78) were overexpressed in HCC tissues. The expression of HSP27 correlated with AFP levels whereas upregulation of GRP78 was associated with tumour venous infiltration, although the association of HSP70 with any pathologic features was not significant [11]. On the other hand, a review by Qin et al. concluded that HSP70 was closely related to several pathological parameters associated with tumour progression, indicating that HSP70 overexpression is not only a marker of hepatocarcinogensis but also a marker of tumour progression and tumour cell proliferation $[3,12]$.

Based on the vital role of HSPs in HCC and controversies in the reported data, further analysis to clarify this relationship between HSPs and HCC prognosis is urgently needed. This study set out to define the relationships between HSPs and outcomes in HBV-related HCC patients, in the hope that the data may provide novel biomarker candidates as well as useful insights into the pathogenesis and progression of HCC.

\section{Methods}

\section{Patients}

A total of 247 patients with HCC were identified. Data on HSP gene expression could not be obtained for 22 of these and a further 5 had insufficient clinical outcome data available, leading to 220 patients being included in the analysis. Cases consisted of patients with a history of hepatitis B virus (HBV) infection or HBV-related liver cirrhosis; the diagnosis of HCC was made in all cases by two independent pathologists who had detailed information on clinical presentation and pathological characteristics.

All liver tissue was obtained with informed consent from patients who underwent radical resection between 2002 and 2003 at the Liver Cancer Institute and Zhongshan Hospital (Fudan University). The study was approved by the Institutional Review Board of the participating institutes [13]. All participants provided written informed consent, as reported by Roessler et al. [13, 14].

\section{Source of data}

We extracted the GSE14520 microarray expression profile from Gene Expression Omnibus (GEO, http://www.ncbi.nlm.nih.gov/geo/) database. Tumour sample and microarray processing were reported by Roessler et al. $[13,14]$ and are available at http://www.ncbi.nlm.nih.gov/geo/query/acc.cgi?ac $\mathrm{c}=\mathrm{GSE} 14520$. HSP gene expression levels were calculated using the matchprobes package in the $\mathrm{R}$ programming environment and the log2 RMA-calculated signal intensity was reported. Details of the experiment protocols and data processing are available at http://www.ncbi.nlm.nih.gov/geo/query/acc.cgi?ac c=GSM362949. We restricted our search to genes within the HSP family, and 25 HSPs were included in our analysis.

\section{End points}

The primary outcome of overall survival was defined as the time from surgery to death from any disease. The secondary outcome of HCC recurrence was defined by: (1) new lesions found in the CT or MRI scans and (2) an abnormal alpha-fetoprotein (AFP) value with a cut-off of $300 \mathrm{ng} / \mathrm{ml}$; including instances when the high pretreatment AFP value did not decrease to a normal level or increased again after becoming normal. 


\section{Statistical analysis}

Parametric data were expressed as mean \pm standard deviation (SD) or median values. The Kolmogorov-Smirnov test was used as a test of normality. Student's $t$-test was used to compare means for normally distributed continuous data, the Mann-Whitney $U$-test was used for non-normally distributed continuous data and the Chi-squared test was used for categorical variables. Factors associated with the outcomes were assessed by univariate analysis and multivariate analysis using Cox regression. Only covariates significantly associated with outcomes according to the univariate analysis (two-sided $P$-value $<0.10)$ are shown and included in the multivariate model. Results were reported as hazard ratios (HR) with 95\% confidence intervals (CI). The Kaplan-Meier method was used to compare overall survival between different groups, and the log-rank test was used to estimate the difference in survival. Statistical analyses were performed using PASW Statistics software version 18.0 from SPSS Inc. (Chicago, IL, USA). All statistical tests were two-tailed, and differences with $P<0.05$ were considered statistically significant.

\section{Results}

\section{Patient characteristics}

As shown in Table 1, of the 220 patients, there were 190 males and 30 females with a mean age of $50.8 \pm 10.6$ years. There were 56 patients with evidence of active viral replication, 155 patients were chronic carriers and no information was available for 9 patients. There were 91 patients with an alanine aminotransferase level over $50 \mathrm{U} / \mathrm{L}$ and 80 patients with a main tumour size over $5 \mathrm{~cm}$. There were 44 patients with multinodular tumours and 202 patients with a history of cirrhosis. There were 99 patients with an alpha-fetoprotein level more than $300 \mathrm{ng} / \mathrm{ml}$. There were 93 patients with a TNM staging I, 77 with TNM-II, 48 with TNM-III and 2 with no information available. Most patients obtained Barcelona Clinic liver cancer (BCLC) staging A $(148 / 220)$. There were 97 patients with Cancer of the Liver Italian Program (CLIP) staging 0, 74 with CLIP staging 1,34 with CLIP staging 2, 9 with CLIP staging 3, 3 with CLIP staging 4, 1 with CLIP staging 5 and no information was available for 2 patients.

\section{HSP expression levels}

HSP expression levels between tumour and non-tumour tissues from HCC patients are shown in Table 2. HSPA4L, HSPA12A and HSPB8 were similarly expressed between tumour tissues and non-tumour tissues from HCC patients $(P=0.620$,
0.895 and 0.168 , respectively). With the exception of HSPA4L, HSPA12A and HSPB8, members of the HSP family, including HSPH1, HSPBP1, HSPA1A, HSPA1B, HSPA1L, HSPA2, HSPA4, HSPA5, HSPA8, HSPA9, HSPAA1, HSPAB1, HSPA14, HSPB11, HSPA13, HSP90B1 and HSPBAP1 were all overexpressed in tumour tissues (all $P<0.001$ ). In contrast, HSPB6, HSPB7, HSPA6, HSPB2 and HSPB3 were more highly expressed in non-tumour tissues compared with tumour tissues from HCC patients (all $P<$ $0.001)$.

Table 1. Baseline characteristics of hepatocellular carcinoma patients.

\begin{tabular}{ll}
\hline Category & $\mathrm{n} /$ mean \pm SD \\
\hline Gender (male/female) & $190 / 30$ \\
Age (years) & $50.8 \pm 10.6$ \\
HBV viral status (AVR-CC/no/NA) & $56 / 155 / 9$ \\
Alanine aminotransferase (>50/<50/NA), U/L & $91 / 129 / 0$ \\
Main tumour size (>5/<5/NA), cm & $80 / 139 / 1$ \\
Multinodular (yes/no/NA) & $44 / 176 / 0$ \\
Cirrhosis (yes/no/NA) & $202 / 18 / 0$ \\
TNM staging (I/II/III/NA) & $93 / 77 / 48 / 2$ \\
BCLC staging (0/A/B/C/NA) & $20 / 148 / 22 / 28 / 2$ \\
CLIP staging (0/1/2/3/4/5/NA) & $97 / 74 / 34 / 9 / 3 / 1 / 2$ \\
Alpha-fetoprotein (>300/<300/NA), ng/ml & $99 / 119 / 2$ \\
\hline NA, not available; AVR-CC, active viral replication chronic carrier.
\end{tabular}

\section{Factors associated with survival in HCC}

All characteristics and HSPs included in the analysis are summarised in Table 3. Univariate analysis showed that main tumour size over $5 \mathrm{~cm}$, multinodular tumours, cirrhosis, TNM staging, BCLC staging, CLIP staging, AFP level and tumour tissue HSPs (HSPA12A, HSPA1A, HSPA1B, HSPA5, HSP90AB1, HSPA14, HSPB11 and HSP90B1) were all factors associated with overall survival in HCC patients (all $P<0.10$ ). When these factors were evaluated by a multivariate model using forward selection, cirrhosis and BCLC staging were significantly associated with survival $(\mathrm{HR}=5.282,95 \% \mathrm{CI}=1.294-21.555, P=$ 0.020 and $\mathrm{HR}=2.151,95 \% \mathrm{CI}=1.682-2.750, P<0.001$, respectively) and HSPA12A and HSP90B1 were negatively associated with survival of HCC patients (HR $=1.042,95 \% \mathrm{CI}=1.003-1.082, P=0.033$ and $\mathrm{HR}=$ $1.001,95 \% \mathrm{CI}=1.000-1.003, P=0.011$, respectively).

We performed a Kaplan-Meier event analysis, grouping those factors identified to be significantly associated with survival as presented above. As shown in Figure 1. This revealed that the higher the BCLC staging, the greater the risk of death (mean survival time $=64.57,52.49,38.21$ and 24.47 months according to BCLC staging $0, A, B$ and C, respectively; $\log$ rank $P<0.001$, Figure 1A). Cirrhosis also negatively impacted on survival (mean survival time cirrhosis $=47.82$ and no cirrhosis $=63.82$ months, respec- 
tively; $\log$ rank $P=0.019$, Figure 1B). For HSPA12A and HSP90B1, we grouped by median expression into a low expression group and a high expression group. With a median cut-off of 11.88 , high expression of HSPA12A contributed to poorer overall survival in HCC patients (mean survival time high $=45.52$ and low $=52.11$ months, respectively; $\log$ rank $P=0.024$, Figure 1C). Similarly, up-regulation of HSP90B1 in HCC tumour tissues was associated with poor overall survival grouped by median cut-off 819.24 (mean survival time high $=52.85$ and low $=45.12$ months, respectively; $\log$ rank $P=0.032$, Figure 1D).

Table 2. HSPs expression levels between tumour issue and non-tumour issue of HCC patients, [Mean \pm SD / Median (Minimum-Maximum), unit].

\begin{tabular}{|c|c|c|c|c|}
\hline HSPs & HSPs family & Tumour tissues & Non-tumour tissues & $P$ \\
\hline HSPH1 & HSP70 & $122.70(30.09-562.01)$ & $68.61(14.41-523.58)$ & $<0.001$ \\
\hline HSPB6 & Small HSPs & $13.21(9.12-54.66)$ & $15.83(9.95-51.45)$ & $<0.001$ \\
\hline HSPA4L & HSP70 & $21.92(8.85-165.28)$ & $22.72(9.81-134.87)$ & 0.62 \\
\hline HSPBP1 & HSP70 & $42.08(17.02-158.20)$ & $32.25(16.03-63.22)$ & $<0.001$ \\
\hline HSPA12A & HSP70 & $11.88(8.18-37.46)$ & $11.93(9.10-50.88)$ & 0.895 \\
\hline HSPB8 & Small HSPs & $17.44(9.26-1743.44)$ & $16.56(9.40-328.73)$ & 0.168 \\
\hline HSPB7 & Small HSPs & $12.73(9.52-198.27)$ & $13.79(9.42-52.20)$ & $<0.001$ \\
\hline HSPA1A & HSP70 & 1978.667 (319.08-7201.75) & $1317.11(75.95-4639.60)$ & $<0.001$ \\
\hline HSPA1B & HSP70 & $161.39(11.23-1551.73)$ & 77.19 (11.86-1134.17) & $<0.001$ \\
\hline HSPA1L & HSP70 & $14.64(10.50-50.98)$ & $13.30(9.76-24.23)$ & $<0.001$ \\
\hline HSPA2 & HSP70 & $28.47(10.64-1101.85)$ & $20.11(9.77-168.25)$ & $<0.001$ \\
\hline HSPA4 & HSP70 & $163.63(33.78-557.95)$ & $67.03(17.36-191.30)$ & $<0.001$ \\
\hline HSPA5 & HSP70 & 3186.59 (629.95-7980.99) & $1976.33(68.30-4980.05)$ & $<0.001$ \\
\hline HSPA6 & HSP70 & $21.70(10.12-204.35)$ & $34.14(11.75-320.93)$ & $<0.001$ \\
\hline HSPA8 & HSP70 & $2826.45 \pm 1132.50$ & $1794.61 \pm 726.08$ & $<0.001$ \\
\hline HSPA9 & GRP75 & $228.63(20.46-803.17)$ & $170.49(13.74-262.98)$ & $<0.001$ \\
\hline HSPB2 & Small HSPs & $12.96(8.44-90.53)$ & $15.21(9.18-43.48)$ & $<0.001$ \\
\hline HSP90AA1 & HSP90 & $1940.99(228.72-5086.35)$ & $1128.81(33.42-2579.93)$ & $<0.001$ \\
\hline HSP90AB1 & HSP90 & $832.94(94.56-3305.60)$ & $348.03(15.04-992.15)$ & $<0.001$ \\
\hline HSPA14 & HSP70 & $80.45(12.10-265.52)$ & $32.32(13.82-85.22)$ & $<0.001$ \\
\hline HSPB11 & Small HSPs & $132.74(27.93-1246.39)$ & $80.67(16.56-158.26)$ & $<0.001$ \\
\hline HSPA13 & HSP70 & $50.59(14.50-340.89)$ & $20.59(10.28-76.52)$ & $<0.001$ \\
\hline HSP90B1 & HSP90 & 819.24 (115.46-2409.27) & $584.01(48.40-1164.86)$ & $<0.001$ \\
\hline HSPBAP1 & Small HSPs & $49.33(12.29-167.42)$ & $38.68(14.79-100.58)$ & $<0.001$ \\
\hline HSPB3 & Small HSPs & $11.30(8.53-115.97)$ & $12.03(9.23-47.80)$ & $<0.001$ \\
\hline
\end{tabular}

Figure 1. Overall survival analysis of HBV-related $\mathrm{HCC}$ patients of $(A) B C L C$ staging, (B) cirrhosis, (C) HSPA12A with a 11.88 cut-off, and (D) HSP90B1 with a 819.24 cut-off by Kaplan-Meier survival method.
A

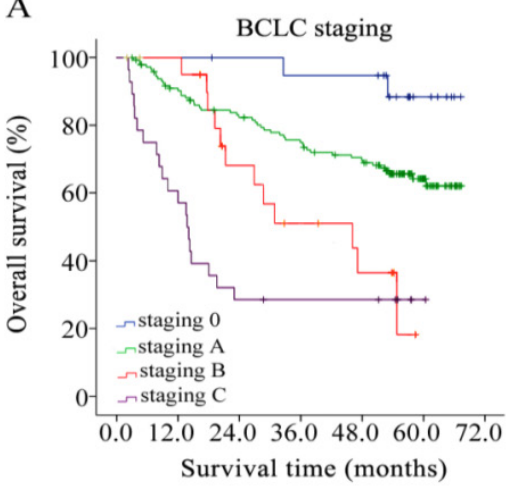

$\mathrm{C}$

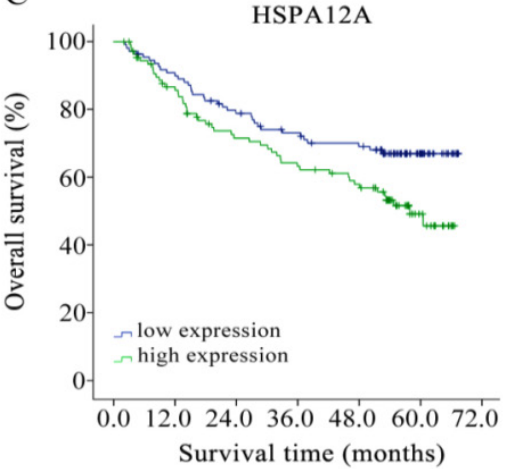

B

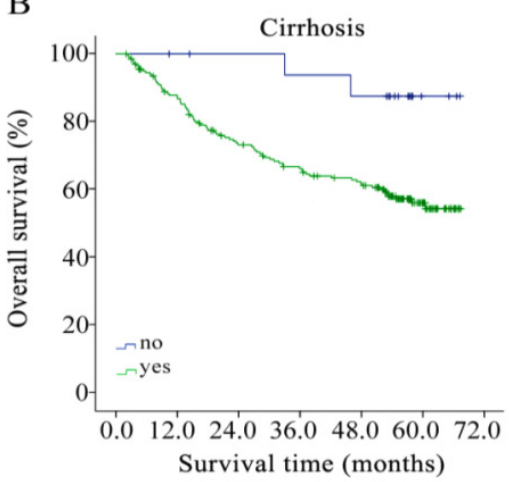

D

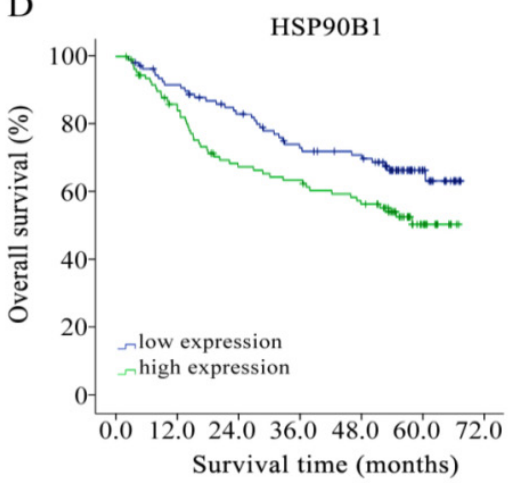




\section{Factors associated with recurrence of HCC}

Table 4 summarises the results from the univariate and multivariate regression analyses of risk factors associated with recurrence of HCC in patients. Male sex, main tumour size greater than $5 \mathrm{~cm}$, cirrhosis, TNM staging, BCLC staging, CLIP staging and tumour tissue HSPs (HSPH1, HSPA4L, HSPA4, HSPA5, HSPA6 and HSP90B1) were all factors associated with recurrence of HCC (all $P<0.10$ ). Furthermore, multivariate analysis using forward selection showed that high BCLC staging was a risk factor associated with relatively earlier recurrence of HCC in patients $(\mathrm{HR}=1.797,95 \% \mathrm{CI}=1.439-2.244, P<0.001)$. Interestingly, HSP70 family members including HSPA4, HSPA5 and HSPA6 were significantly associated with $\mathrm{HCC}$ recurrence $(\mathrm{HR}=1.002,95 \% \mathrm{CI}=$ 1.000-1.004, $P=0.019 ; \mathrm{HR}=1.0,95 \% \mathrm{CI}=1.0-1.0, P=$ 0.046 and $\mathrm{HR}=1.008,95 \% \mathrm{CI}=1.001-1.015, P=0.021$, respectively).

\section{Discussion}

HSPs are a set of highly conserved proteins whose expression is induced in response to anti-cancer chemotherapy, thus allowing the cell to survive to lethal conditions. Cancer cells experience high levels of proteotoxic stress and rely upon stress-response pathways for survival and proliferation, thereby becoming dependent on proteins such as stress-inducible HSPs. The major HSPs in mammalian cells have been classified into four main families according to their molecular weight: HSP90, HSP70, HSP60 and small HSPs (15-30 kDa) which includes HSP27.

In our analysis, even though HSPA4L (heat shock $70 \mathrm{kDa}$ protein $4 \mathrm{~L}$ ), HSPA12A (heat shock 70 $\mathrm{kDa}$ protein 12A) and HSPB8 (HSP22) were similarly expressed in both tumour and non-tumour tissues of HCC patients, most of the HSPs including HSP60 (HSPA14), HSP70 (HSPH1, HSPBP1, HSPA1A, HSPA1B, HSPA1L, HSPA2, HSPA4, HSPA5, HSPA8, HSPA9, HSPA13), HSP90 (HSP90AA1, HSP90AB1, HSP90B1) and small HSPs (HSPB11, HSPBAP1) were expressed at higher levels in HCC tumour tissues. Increased HSP expression in tumour tissues is a common phenomenon and the potential reasons for this have been summarised elsewhere [15]. However, most small HSPs (HSPB2, HSPB3, HSPB6, HSPB7) and HSPA6 were upregulated in HCC non-tumour tissues. Univariate and multivariate regression analyses showed that HSPA6 was a risk factor for HCC recurrence, which warrants further research.

Table 3. Cox regression analysis of risk factors associated with survival of HCC patients.

\begin{tabular}{|c|c|c|c|c|}
\hline Covariates & Univariate analysis, $\mathrm{HR}(95 \% \mathrm{CI})$ & $P$ & Multivariate Analysis, HR (95\% CI) & $P$ \\
\hline Main tumour size $(>5 \mathrm{~cm}$ vs. $<5 \mathrm{~cm})$ & $1.969(1.279-3.032)$ & 0.002 & & \\
\hline Multinodular (yes vs. no) & $1.693(1.047-2.738)$ & 0.032 & & \\
\hline Cirrhosis (yes vs. no) & $4.576(1.125-18.613)$ & 0.034 & $5.282(1.294-21.555)$ & 0.02 \\
\hline TNM staging, per increase of 1 level & $2.263(1.704-3.004)$ & $<0.001$ & & \\
\hline BCLC staging, per increase of 1 level & $2.143(1.688-2.722)$ & $<0.001$ & $2.151(1.682-2.75)$ & $<0.001$ \\
\hline CLIP staging, per increase of 1 level & $1.901(1.536-2.353)$ & $<0.001$ & & \\
\hline AFP (>300ng/ml vs. <300ng/ml) & $1.598(1.041-2.455)$ & 0.032 & & \\
\hline HSPA12A, per increase of 1 unit & $1.038(1.002-1.076)$ & 0.036 & $1.042(1.003-1.082)$ & 0.033 \\
\hline HSPA1A, per increase of 1 unit & $1.0(1.0-1.0)$ & 0.065 & & \\
\hline HSPA1B, per increase of 1 unit & $1.001(1.0-1.002)$ & 0.01 & & \\
\hline HSPA5, per increase of 1 unit & $1.0(1.0-1.0)$ & 0.001 & & \\
\hline HSP90AB1, per increase of 1 unit & $1.0(1.0-1.001)$ & 0.061 & & \\
\hline HSPA14, per increase of 1 unit & 1.005 (1.001-1.009) & 0.018 & & \\
\hline HSPB11, per increase of 1 unit & $1.001(1.0-1.003)$ & 0.078 & & \\
\hline HSP90B1, per increase of 1 unit & $1.001(1.0-1.001)$ & 0.009 & $1.001(1.0-1.001)$ & 0.011 \\
\hline
\end{tabular}

Table 4. Cox regression analysis of risk factors associated with recurrence of HCC patients.

\begin{tabular}{|c|c|c|c|c|}
\hline Covariates & Univariate analysis, HR (95\%CI) & $P$ value & Multivariate Analysis, HR (95\% CI) & $P$ value \\
\hline Gender (male vs. female) & $2.15(1.125-4.109)$ & 0.021 & & \\
\hline Main tumour size $(>5 \mathrm{~cm}$ vs. $<5 \mathrm{~cm}$ ) & $1.414(0.977-2.044)$ & 0.066 & & \\
\hline Cirrhosis (yes vs. no) & $2.169(0.954-4.93)$ & 0.065 & & \\
\hline TNM staging, per increase of 1 level & $1.765(1.401-2.223)$ & $<0.001$ & & \\
\hline BCLC staging, per increase of 1 level & $1.791(1.45-2.212)$ & $<0.001$ & $1.797(1.439-2.244)$ & $<0.001$ \\
\hline CLIP staging, per increase of 1 level & $1.461(1.216-1.757)$ & $<0.001$ & & \\
\hline HSPH1, per increase of 1 unit & $1.002(1.0-1.004)$ & 0.065 & & \\
\hline HSPA4L, per increase of 1 unit & $1.006(1.0-1.012)$ & 0.068 & & \\
\hline HSPA4, per increase of 1 unit & $1.002(1.0-1.003)$ & 0.085 & $1.002(1.0-1.004)$ & 0.019 \\
\hline HSPA5, per increase of 1 unit & $1.0(1.0-1.0)$ & 0.008 & $1.0(1.0-1.0)$ & 0.046 \\
\hline HSPA6, per increase of 1 unit & $1.007(1.0-1.014)$ & 0.055 & $1.008(1.001-1.015)$ & 0.021 \\
\hline HSP90B1, per increase of 1 unit & $1.0(1.0-1.001)$ & 0.041 & & \\
\hline
\end{tabular}

HR, hazard ratios; CI, confidence interval. 
Our data demonstrated that the overexpression of HSPA12A in HCC tumour tissues was significantly related to poor survival of HCC patients. HSPA12A is a novel and atypical member of the HSP70 family in animals. Its effects are diverse and there have been reported involvement in atherosclerotic lesion development in mice [17], mediation of immune responses and environmental stress in mussels [18], association with muscular function in Japanese black cattle [19] and the development of mouse palates through participating in the stress-response process and/or the antiapoptosis process [20]. It has been reported that HSP70 could serve as a molecular marker for early HCC [21, 22]. For instance, Chuma et al. [23] compared the expression profiles between seven early components and seven progressed components of nodule-in-nodule-type HCCs and their corresponding noncancerous liver tissues. Of the 95 genes, the most abundantly upregulated gene in early HCC components was HSP70. Further immunohistochemical examination of HSP70 revealed its significant overexpression in early HCC compared with precancerous lesions and also in progressed HCC compared with early HCC. Hence, HSPA12A could be a candidate for a novel biomarker for HCC. We report that HSPA12A was similarly expressed both in HCC tumour and non-tumour tissues and further research should focus on understanding the mechanisms by which HSPA12A influences HCC pathogenesis and progression.

As a member of the HSP90 family (also known as GRP94 and gp96), HSP90B1 is associated with cancer metastasis and decreased survival and thus constitutes a relevant therapeutic target in various types of cancer [24-26]. Being one of the proteins residents in the endoplasmic reticulum, the immunological properties of HSP90B1, as well as the cytosolic proteins HSP70 and HSP90, have been studied intensively. A series of animal studies [27, 28] documented that HSP90B1 purified from tumours was able to initiate efficient tumour specific cytotoxic T-lymphocytic responses. Moreover, HSP90B1 works in both prophylactic and therapeutic protocols. Meanwhile, an autologous HSP90B1 vaccine treatment seems to be a well-tolerated therapeutic option [29,30]. Experiments revealed that HSP90B1 and HSP70 were equally immunogenic and considerably superior to HSP90, the cytoplasmic analogue of HSP90B1 [31]. Meng et al. $[32,33]$ reported the identification of a HBV-specific, HLA class I-specific peptide bound to HSP90B1 in HCC patients, indicating its possible role in immunogenicity of HBV-induced HCC patients. A study by Tanaka et al. [34] showed that HSP90B1 was preferentially accumulated in the nuclei of HCC cells and implied its effect on tumorigenicity. With an experi- ment aimed to test the correlation between HSP90B1 expression level and HBV-induced disease progression, Zhu et al. [35] found that the extent of elevated HSP90B1 expression was higher in HCC patients, lower in chronic HBV infection and was of medium expression in cirrhosis patients. Moreover, Lim et al. [6] performed a study revealing that there was a positive correlation between the expression of HSP90B1 and prognostic factors of HCC. Specifically, the expression of HSP90B1 was associated significantly with vascular invasion and intrahepatic metastasis. In our analysis, HSP90B1 showed significant correlation with HCC survival; thus, the potential for using HSP90B1 in HCC diagnostics and therapeutics warrants investigation.

Upregulation of HSP70 protein in HCC may be functionally related to tumour progression. However, it is of great importance to further define the subtypes of HSP70, which may also be used as a novel diagnostic and prognostic marker for HCC. In our study, HSPA4, HSPA5 and HSPA6 overexpression significantly associated with recurrence of HCC. HSPA4 was overexpressed in as many as $78 \%$ of HCC tumour tissues. Because no relationship has previously been shown between HSPA4 and tumour metastasis or transformation, it is intriguing that siRNA mediated repression of HSPA4 has been reported to cause a significant decrease in in vitro migration, invasion and transformation activity [36,37], which supports our observation. It is well known that HSPA5 promotes the invasion of HCC, is associated with poor survival of HBV-related HCC patients and is a potential target for inhibiting the invasion of HCC cells $[6,38,39]$, which are also consistent with our analyses. Despite being a member of the HSP70 family, few studies have evaluated the role of HSPA6 in HCC patients. Intriguingly, our results showed that HSPA6, overexpressed in HCC non-tumour tissues, may be a potential biomarker in predicting the recurrence of $\mathrm{HCC}$, although further research is required. Phylogenetic analysis indicated that the HSPA6 protein sequence was closely related to HSP72, another major inducible human HSP70. The roles of HSP70 and HSP72 in HCC have been evaluated intensively $[6,7,11,40]$, but our results suggest that further approaches should be taken to consider the role of HSPA6 in the progression of HCC. We have demonstrated some strong associations, which indicate that further studies looking at potential mechanisms are required.

Of the clinical parameters investigated, we showed that advanced BCLC staging and a history of cirrhosis are risk factors for HCC patient-related mortality, and that advanced BCLC staging is also significantly associated with recurrence of HCC, which is in keeping with previous reports $[13,41,42]$. 
Moreover, BCLC staging also contributed to relatively earlier recurrence of early-stage HCC patients. Hence, clinical oncologists should consider evaluating the role of BCLC staging when forming prognoses for early-stage HCC patients. It has been reported that patients with cirrhosis are at the highest risk for developing HCC and that this is associated with poor survival $[3,43]$, which is in keeping with our findings.

This study has two main limitations: First, this study was based on data from a national data bank, and no direct first-hand data were available. Second, we included HSP expression as a continuous variable in the Cox regression process, therefore the HRs of the HSP candidate markers were small. Even with small HRs for these HSP sub-families, the results might provide insights for further research.

In conclusion, most HSPs are more highly expressed in tumour than non-tumour tissues. The overexpression of HSPA12A and HSP90B1 should be associated with poor survival from HCC, whereas higher levels of HSPA4, HSPA5 and HSPA6 might relate to earlier recurrence of HCC. Further research could evaluate the utility of these HSPs in HCC prevention, therapeutics and prognostics.

\section{Acknowledgement}

This work was supported by the National Science and Technology Major Projects of the Twelfth Five-year Plan (2012ZX10004301004). The funder had no role in study design, data collection and analysis, decision to publish, or preparation of the manuscript.

\section{Abbreviations}

HSPs, heat shock proteins; GRP, glucose-regulated protein; HBV, hepatitis B virus; HCC, hepatocellular carcinoma; AFP, alpha-fetoprotein; GEO, Gene Expression Omnibus; HR, hazard ratios; $\mathrm{CI}$, confidence interval.

\section{Competing Interests}

The authors have declared that no competing interest exists.

\section{References}

1. Ferlay J, Bray F, Pisani P, Parkin DM. GLOBOCAN 2002: Cancer incidence, mortality and prevalence worldwide IARC. (Cancer Base No.5 Version 2.0). Lyon, France: IARCPress. 2004.

2. Parkin DM. Global cancer statistics in the year 2000. Lancet Oncol 2001,2(9):533-543.

3. Shariff MI, Cox IJ, Gomaa AI, Khan SA, Gedroyc W, Taylor-Robinson SD. Hepatocellular carcinoma: current trends in worldwide epidemiology, risk factors, diagnosis and therapeutics. Expert Rev Gastroenterol Hepatol 2009,3(4):353-367.

4. Siegel R, Naishadham D, Jemal A. Cancer Statistics, 2012. CA Cancer J Clin 2012,62:10-29

5. Yao DF, Dong ZZ, Yao M. Specific molecular markers in hepatocellular carcinoma. Hepatobiliary Pancreat Dis Int 2007,6(3):241-247.

6. Lim SO, Park SG, Yoo JH, et al. Expression of heat shock proteins (HSP27, HSP60, HSP70, HSP90, GRP78, GRP94) in hepatitis B virus-related hepatocellular carcinomas and dysplastic nodules. World J Gastroenterol 2005,11(14):2072-2079.
7. Cui N, Xu YP, Cao ZH, Xu FX, Zhang P, Jin LJ. Effects of heat stress on the level of heat shock protein 70 on the surface of hepatocellular carcinoma Hep G2 cells: implications for the treatment of tumors. Tumor Biol 2013,34(2):743-8.

8. Cawthorn TR, Moreno JC, Dharsee M, et al. Proteomic analyses reveal high expression of decorin and endoplasmin (HSP90B1) are associated with breast cancer metastasis and decreased survival. PLoS One 2012,7(2):e30992.

9. Jego G, Hazoumé A, Seigneuric R, Garrido C. Targeting heat shock proteins in cancer. Cancer Lett 2013,332(2):275-85.

10. Ciocca DR, Cayado-Gutierrez N, Maccioni M, Cuello-Carrion FD. Heat Shock Proteins (HSPs) Based Anti-Cancer Vaccines. Curr Mol Med 2012,12(9):1183-97.

11. Luk JM, Lam CT, Siu AFM, et al. Proteomic profiling of hepatocellular carcinoma in Chinese cohort reveals heat-shock proteins (Hsp27, Hsp70, GRP78) pregulation and their associated prognostic values. Proteomics 2006,6:1049-1057.

12. Qin LX, Tang ZY. Recent progress in predictive biomarkers for metastatic recurrence of human hepatocellular carcinoma: a review of the literature. J Cancer Res Clin Oncol 2004,130:497-513.

13. Roessler S, Jia HL, Budhu A, et al. A Unique Metastasis Gene Signature Enables Prediction of Tumor Relapse in Early-Stage Hepatocellular Carcinoma Patients. Cancer Res 2010,70(24):10202-12.

14. Roessler S, Long EL, Budhu A, et al. Integrative genomic identification of genes on $8 \mathrm{p}$ associated with hepatocellular carcinoma progression and patient survival. Gastroenterology 2012,142(4):957-966.

15. Calderwood SK, Khaleque MA, Sawyer DB, Ciocca DR. Heat shock proteins in cancer: chaperones of tumorigenesis. Trends Biochem Sci 2006,31(3):164-72

16. Chen YC, Lin-Shiau SY, Lin JK. Involvement of heat-shock protein 70 and p53 proteins in attenuation of UVC-induced apoptosis by thermal stress in hepatocellular carcinoma cells. Photochem Photobiol 1999,70(1):78-86.

17. Han ZH, Truong QA, Park S, Breslow JL. Two Hsp70 family members expressed in atherosclerotic lesions. Proc Natl Acad Sci USA 2003,100(3):1256-61.

18. You LP, Ning XX, Liu F, Zhao JM, Wang Q, Wu HF. The response profiles of HSPA12A and TCTP from Mytilus galloprovincialis to pathogen and cadmium challenge. Fish Shellfish Immunol 2013,35(2):343-50.

19. Masoudi AA, Uchida K, Yokouchi $K$, et al. Linkage mapping of the locus responsible for forelimb-girdle muscular anomaly of Japanese black cattle on bovine chromosome 26. Anim Genet 2008,39(1):46-50.

20. Zhu YF, Ren CL, Wang XY, et al. Gene expression of Hsp70, Hsp90 and Hsp110 families in normal palate and cleft palate during mouse embryogenesis. Toxicol Ind Health 2013,29(10):915-30.

21. Effendi K, Sakamoto M. Molecular pathology in early hepatocarcinogenesis. Oncology 2010,78(2):157-60.

22. Sakamoto M, Mori T, Masugi Y, Effendi K, Rie I, Du W. Candidate molecular markers for histological diagnosis of early hepatocellular carcinoma. Intervirology 2008,51(Suppl 1):42-5.

23. Chuma M, Sakamoto M, Yamazaki K, et al. Expression profiling in multistage hepatocarcinogenesis: identification of HSP70 as a molecular marker of early hepatocellular carcinoma. Hepatology 2003,37(1):198-207.

24. Workman P, Burrows F, Neckers L, Rosen N. Drugging the cancer chaperone HSP90: combinatorial therapeutic exploitation of oncogene addiction and tumor stress. Ann N Y Acad Sci 2007,1113:202-216.

25. Li GD, Cai M, Fu D, et al. Heat shock protein 90B1 plays an oncogenic role and is a target of microRNA-223 in human osteosarcoma. Cell Physiol Biochem 2012,30(6):1481-90

26. Cawthorn TR, Moreno JC, Dharsee $M$, et al. Proteomic analyses reveal high expression of decorin and endoplasmin (HSP90B1) are associated with breast cancer metastasis and decreased survival. PLoS One 2012,7(2):e30992.

27. Yedavelli SP, Guo L, Daou ME, Srivastava PK, Mittelman A, Tiwari RK. Preventive and therapeutic effect of tumor derived heat shock protein, gp96, in an experimental prostate cancer model. Int J Mol Med 1999,4(3):243-8.

28. Kovalchin JT, Murthy AS, Horattas MC, Guyton DP, Chandawarkar RY. Determinants of efficacy of immunotherapy with tumor-derived heat shock protein gp96. Cancer Immun 2001,1:7-15.

29. Arnold-Schild D, Kleist C, Welschof M, et al. One-step single-chain Fv recombinant antibody-based purification of gp96 for vaccine development. Cancer Res 2000, 60(15):4175-8

30. Randazzo M, Terness P, Opelz G, Kleist C. Active-specific immunotherapy of human cancers with the heat shock protein Gp96-revisited. Int J Cancer 2012,130(10):2219-31.

31. Udono H, Srivastava PK. Comparison of tumor-specific immunogenicities of stress-induced proteins gp96, hsp90, and hsp70. J Immunol 1994,152(11):5398-403.

32. Meng SD, Gao T, Gao GF, Tien P. HBV-specific peptide associated with heat-shock protein gp96. Lancet 2001,357(9255):528-9.

33. Meng SD, Song J, Rao Z, Tien P, Gao GF. Three-step purification of gp96 from human liver tumor tissues suitable for isolation of gp96-bound peptides. J Immunol Methods 2002,264(1-2):29-35.

34. Tanaka K, Kondoh N, Shuda M, et al. Enhanced expression of mRNAs of antisecretory factor-1, gp96, DAD1 and CDC34 in human hepatocellular carcinomas. Biochim Biophys Acta 2001,1536(1):1-12.

35. Zhu XD, Li CL, Lang ZW, Gao GF, Tien P. Significant correlation between expression level of HSP gp96 and progression of hepatitis B virus induced diseases. World J Gastroenterol 2004,10(8):1141-5. 
36. Yang MH, Chang SY, Chiou SH, et al. Overexpression of NBS1 induces epithelial-mesenchymal transition and co-expression of NBS1 and Snail predicts metastasis of head and neck cancer. Oncogene 2007,26(10):1459-67.

37. Wu CY, Lin CT, Wu MZ, Wu KJ. Induction of HSPA4 and HSPA14 by NBS1 overexpression contributes to NBS1-induced in vitro metastatic and transformation activity. J Biomed Sci 2011,18:1.

38. Su RJ, Li Z, Li HD, et al. Grp78 promotes the invasion of hepatocellular carcinoma. BMC Cancer 2010,10:20.

39. Li HD, Song HJ, Luo JS, Liang J, Zhao S, Su RJ. Knockdown of glucose-regulated protein 78 decreases the invasion, metalloproteinase expression and ECM degradation in hepatocellular carcinoma cells. J Exp Clin Cancer Res 2012,31:39.

40. Wang $X P$, Zhou $Y X$, Sun LJ, et al. Complex formation between heat shock protein 72 and hepatitis $B$ virus $X$ protein in hepatocellular carcinoma tissues. J Proteome Res 2008,7(12):5133-7.

41. Kim BK, Kim SU, Park JY, et al. Applicability of BCLC stage for prognostic stratification in comparison with other staging systems: single centre experience from long-term clinical outcomes of 1717 treatment-naïve patients with hepatocellular carcinoma. Liver Int 2012,32(7):1120-7.

42. Guglielmi A, Ruzzenente A, Pachera S, et al. Comparison of seven staging systems in cirrhotic patients with hepatocellular carcinoma in a cohort of patients who underwent radiofrequency ablation with complete response. Am J Gastroenterol 2008,103(3):597-604.

43. Hernandez-Gea V, Toffanin S, Friedman SL, Llovet JM. Role of the microenvironment in the pathogenesis and treatment of hepatocellular carcinoma. Gastroenterology 2013,144(3):512-27. 